\section{LUSTRUM, Internationale Forschungsberichte aus dem Bereich des klassischen Altertums, Göttingen}

\section{SILVIA MILANEZI \\ Université Pierre Mendès France \\ Grenoble II}

Lustrum fête cette année quarante ans d'existence $^{1}$. Cette revue indispensable à tout travail de recherche sur l'Antiquté classique est née à la suite du Ile Congrès International d'Études Classiques, tenu à Copenhagen en août 1954, en replacement à la revue Bursianschen Jahresberichte. On forma alors une association internationale, sous la direction de Hans Joachim METTE et Andreas THIERFELDER ${ }^{2}$, qui se donnait pour but la publication annuelle de la nouvelle revue bibliographique, Lustrum, subventionnée par I'UNESCO et par des centres de recherches nationaux. Les articles de bibliographie critique, confiés à spécialistes reconnus dans les principaux domaines de l'Antiquité Classique, devaient prendre en compte les progrés réalisés dans telle ou telle discipline dans les cinq dernières années. On prévoyait cependant que les responsables des articles recensent également les études critiques publiées les décennies antérieures au cas où une bibliographie raisonnée sur auteur ou sur une question donnée ne serait pas disponible. Ainsi nous retrouvons dans le premier volume un article sur les rapports entre l'archéologie et la littérature qui couvre un lustre, tandis que l'article sur Homère s'occupe de trois décennies ${ }^{3}$. Les éditeur-fondateur décidaient de laisser de côté les domaines de l'antiquité classique couverts par d'autre publications spécialisées comme la linguistique et l'archéologie (même si on les évoquait quand le besoin se faisait sentir en rapport avec d'autre disciplines ${ }^{4}$ ) pour s'occuper davantage des auteurs anciens, voire des genres littéraires au sens large qu'ils représentent (poésie, histoire, rhétorique, philosophie) et des disciplines parfois délaissées par les spécialistes comme la musique ${ }^{5}$ ou la métrique ${ }^{6}$. Les langues utilisées seraiẹnt le latin, l'allemand, le français, l'anglais er l'italien.

Dès le premier numéro, METTE et THIERFELDER, rendaient hommenage à l'Année Philologique, instrument de travail par excellence pour tous ceux qui s'intéressent à l'Antiquité Classique, qu'ils soient des chercheurs confirmés ou des débutants. En effect, Lustrum, ne se présentait pas comme un concurrent de cette célèbre institution. Tandis que l'Année Philologique recense annuellement presque la totalité des études concernant l'Antiquité Classique, Lustrum proposerait une étude critique de cette bibliographie, tout en gardant les indications, voir les abréviations de l'Année Philologique ${ }^{7}$. Prenons comme exemple l'article de H. J. METTE, Homer 1930$1956^{8}$. Après une présentation sur la question, l'auteur divise son travail en dix-sept chapitres répartis de la façon suivante: Édition du texte; scholies; commentaires; métrique; langue, scènes typiques, épithètes, formules, héros homérique, sociologie et discours; religion; nature et paysage; archéologie; Homère et l'Orient; les ébauches de la poésie épique; la poésie épique elle-même; l'héritage homérique, Homère, le poète. Ces sections débutent et se renferment souvent par un rapide commentaire du spécialiste qui peut en quelques lignes rendre compte des ouvrages ou des articles le plus remarquables, voire les indispensables sur la question ou simplesment les signales en les numérotant. Cette numérotation est plus tard reprise dans un index très maniable qui permet au chercheur de se repérer rapidement et efficacement. Dans ces sections il ne faut jamais s'attendre à ce que les articles répertoriés soient présentés selon l'ordre alphabétique. Ces principes de base se retrouvent dans la plupart des articles publiés chez Lustrum - qu'ils occupent quelques dizaines de pages ou le volume tout entier ${ }^{9}$ - et cela même après la 
disparition de H. J. METTE et de A. THIERFELDER ${ }^{10}$.

Dans un article de 1977, Luc BRISSON affirmait: "Le phénomène majeur dans le domaine de la recherche, ces dernières années, réside dans ce qu'il faut appeler la saturation. Sur qelque sujet que ce scit, le nombre des travaux augmente au point que ce qui devait servir d'intermédiaire entre un objet et celui que s'y intéresse se transforme en écran. Et cette inflation, loin de se réduire s'accrôt à un rythme accéléré"11. Nous pourrions nuancer cette affirmation de Luc Brisson et nous réjouir de l'intérêt que les mondes anciens semblent susciter chez les nouvelles générations. Cependant, il a raison de souligner les difficultés du chercheur face aux nouvelles et innombrables publications. Sa réflexion et notre expérience confirment qu'une revue comme Lustrum reste un instrument de travail irremplaçable, indispensable vie à cette revue à l'occasion de cet anniversaire. Qu'elle soit imprimée sur papier, qu'à l'avenir elle soit informatisée, Lustrum continuera de réfléchir sur publications concernant l'Antiquité Classique, elle continuera de démêler de nombreux écheveaux pour nous permettre de parcurrir plus aisément les labyrinthes de nos disciplines.

\section{Notes}

1 - Lustrum, 1, 1956 (publiée à Göttingen par Vandenhoeck \& Ruprecht et parue en 1957).

2 - Cf. la préface de METTE, H. et THIERFELDER, A. - Eustrum, 1, 1956, p. 5

3 - Cf. WEBSTER, Th. B. L. Greek Archaeology and Literature (1951-1955). Lustrum, 1, 1956 et METTE, H. J. Homer 1930-1956. Lustrum, 1, 1956, p. 7-86.

4 - Cf. WEBSTER, Th: B. L. Greek Archaeology and Literature (1951-1955).Lustrum, 1, 1956, p. 87-120 (voir aussi Lustrum, 6, 1961; 11, 1966; 15, 1970; p. 87-120). Voir aussi HUS A. Sculpture étrusque. Lustrum, 11, 1966, p. 145172.

5 - À propos de la musique, voir WINNINGTON-INGRAM, R. P. Ancient Greek Music 1932-1957. Lustrum, 3, 1958, p. 5-157.
6 - Pour la métrique, voir par exemple: DALE, A. M. Greek Metric 1936-1957. Lustrum, 2, 1957, p. 5-51; HARSH Ph. Wh. Early Latin Meter and Prosody 1904-1955. Lustrum, 3, 1958, p. 215-250; GETTY R. J. Classical Latin Metre and Prosody. Lustrum, 8, 1963, p. 103160. D'autres suites pointus sont traités par cette revue, voir: IRIGOIN J. Les Manuscripts grecs 1931-1960. Lustrum, 7, 1962, p. 5-93; THAER Cl. Antike Mathematik 19311938 (einzelnes auc bis 1943). Lustrum, 6, 1961, p. 38-113; CALBOLI C. I modi del verbo greco e latino 1903-1966. Lustrum, 11, 1966, p. 173-350 (cf. aussi Lustrum, 13, 1968, p. 405-512); COLLART J. Varron grammairien et l'enseignement grammatical dans l'antiquité romaine 1934-1963. Lustrum, 9, 1964, p. 213-242. On y trouve également des articles rares, comme METTE, H. J. Nekrolog einer Epoche: Herman Usener und seine Schule. Ein wirkungsgeschichtlicher Rückblick auf die Jahre 1856-1979. Lustrum, 22, 1979-1980, p. 5-106; surprenants comme ARAI Ts. The Japanese Contribution to Greek and Roman History from the End of the First World War to 1962-1963. Lustrum, 8,1963 , p. $217-243$.

7 - Cf. la préface de METTE, H. J. et THIERFELDER, A. - Lustrum, 1, 1956, p. 6.

8 - Lustrum, 1, 1956, p. 7-86.

9 - Par exemple: METTE, H. J. Euripide (insbesondere für die Jahre 1968-1981). Erster Hauptteil: Die Bruchstücke. Lustrum, 23-24, 1981-1982, p. 5-448 (tout le volume); GRANAROLO, J. Catulle 1960-1985. Lustrum, 2829, 1986-1987, p. 65-106; METTE, H. J. Die "keinen" griechischen Historiker heute. Lustrum, 22, 1979-1980, p. 5-44.

10 - À partir de 1988, Lustrum est publiée sous la direction de Hans GÄRTNER et Hubert PETERSMANN. Rendons hommenage à $\mathrm{H}$. J. METTE et à A. THIERFELDER qui ont accompli un travail titanesque pendant plus de trente ans à la tête de Lustrum. En étudiant l'index de cette revue on remarque que METTE n'a pas cessé de préparer le travail des chercheur à venir en leur proposant des articles bibliographiques d'une très grande qualité et dans des domaines parfois très éloignés les uns des autres (nous citons ici quelques exemples: 
Die rômische Tragödie und die Neufunde zur griechichen Tragödie (insbesondere für die Jahre 1945).Lustrum, 9, 1964, p. 5-212; Homer 1966-1971. Lustrum, 15, 1970; Euripide (insbesondere für die Jahre 1968-1981). Erster Hauptteil: Die Bruchstrücke. Lustrum, 23-24, 1981-1982, p. 5-448; Krates von Pergamon
1953-1983.Lustrum, 26, 1984, p. 94-104; Fragmenta Hesiodea 1967-1984. Lustrum, 27, 1985 , p. 5-22; Philon von Larissa und Antiochos von Askalon, Lustrum, 28-29, 1986-1987, p. 9-63).

11 - Platon 1958-1975.Lustrum, 20, 1977, p. 6. 